\title{
SUMS OF POWERS IN LARGE FINITE FIELDS
}

\author{
CHARLES SMALL
}

\begin{abstract}
If $k$ is a positive integer, then, in any finite field with more than
\end{abstract} $(k-1)^{4}$ elements, every element is a sum of two $k$ th powers.

In this note we prove an "outrageous conjecture" of Kaplansky [3]: for each fixed positive integer $k$, every element of every sufficiently large finite field is a sum of two $k$ th powers. More precisely, we show that every finite field with more than $(k-1)^{4}$ elements is sufficiently large. The proof is a straightforward application of a basic inequality from the theory of diagonal equations over finite fields.

TheOREM. Let $k$ be a positive integer, let $F$ be a finite field with, say, $q$ elements, and put $\delta=(q \cdot-1, k)$. Assume $q>(\delta-1)^{4}$. Then every element of $F$ is a sum of two kth powers. (In particular, the conclusion holds if $q>$ $(k-1)^{4}$, since $k \geqslant \delta$.)

Proof. For $b \in F$ let $N(b)$ denote the number of solutions $(x, y) \in F \times F$ of $x^{k}+y^{k}=b$. Then, by definition, $N(b) \geqslant 0$; we have to show that $q>(\delta-1)^{4}$ implies $N(b)>0$. We may assume $b \neq 0$, since 0 is certainly a sum of two $k$ th powers. Then, by Corollary 1 on p. 57 of [2], we have $|N(b)-q| \leqslant(\delta-1)^{2} \sqrt{q}$. In particular, $N(b)-q \geqslant-(\delta-1)^{2} \sqrt{q}$, so that $N(b)>\sqrt{q}\left(\sqrt{q}-(\delta-1)^{2}\right)$. Hence $N(b)>0$, for all $b$, provided $\sqrt{q}>$ $(\delta-1)^{2}$, or in other words $q>(\delta-1)^{4}$.

The Theorem is best possible in the sense that there are arbitrarily large finite fields in which not everything is a single $k$ th power, for instance the prime fields with $p$ elements, $p \equiv 1(\bmod k)$.

It would be interesting to know if the bound $(\delta-1)^{4}$ is anywhere near best possible. For $k=3,4,5$ the Theorem implies that two $k$ th powers suffice as soon as $q>16,81,256$, respectively, whereas the largest prime fields requiring three $k$ th powers have $7,41,101$ elements, respectively. These computations, as well as the above Theorem in the prime-field case, were noted in [6] and [7].

For $k \leqslant 3$ the Theorem is not new. Nagell [4] showed that the field with seven elements is the only prime field containing elements which are not sums of two cubes. A different proof, based on a theorem of Vosper, appears in [6]. There is also an older proof due to Skolem [5], based on a result of Hurwitz [1]. For arbitrary finite fields $F$ (not necessarily prime), John G. Thompson

Received by the editors September 21, 1976.

AMS (MOS) subject classifications (1970). Primary 12C15; Secondary 14G15.

C American Mathematical Society 1977 
proved (1975; unpublished) by an argument involving group characters that two cubes suffice provided $F$ has more than 25 elements.

\section{REFERENCES}

1. A. Hurwitz, Über die Kongruenz $a x^{e}+b y^{e}+c z^{e} \equiv 0(\bmod p)$, J. Reine Angew. Math. 136 (1909), 272-292.

2. J.-R. Joly, Équations et variétés algébriques sur un corps fini, Enseignement Math. 19 (1973), 1-117. MR 48 \#6065.

3. I. Kaplansky, Private communications, October 7, 1975 and August 25, 1976.

4. T. Nagell, On the solvability of some congruences, Norske Vid. Selsk. Forh. Trondheim 27 (1954), 1-5. MR 16, 220.

5. Th. Skolem, Unlösbarkeit von Gleichungen, deren entsprechende Kogruenz für jeden Modul lösbar ist, Avh. Norske Vid.-Akad. Oslo I 1942, No. 4, 28 pp. MR 8, 7.

6. C. Small, Waring's problem mod n, Amer. Math. Monthly 84 (1977), 12-25.

7. - Solution of Waring's problem mod $n$, Amer. Math. Monthly 84 (1977), 356-359.

Department of Mathematics, Queens University, Kingston, Ontario, Canada 